\title{
HIV AND RISK BEHAVIOUR AMONG MEN WHO HAVE SEX WITH MEN IN SLOVAKIA (2008-2009)
}

Danica Staneková, Patrícia Kramárová, Soňa Wimmerová, Monika Hábeková, Mária Takáčová, Mária Mojzesová Slovak Medical University, Bratislava, Slovakia

\begin{abstract}
SUMMARY
The HIV infection remains a major public health issue in the world and especially in those countries where there exists unprotected sexual intercourse between people of the same sex. It is namely the category of men having sex with men (MSM) that still represents a group at high risk of being infected with HIV or transmitting the virus. The aim of our study was to present HIV 2nd generation surveillance data on MSM in Slovakia in the period between the years 2008 and 2009. Time-location sampling (TLS) was used to recruit participants and oral fluid samples together with completed anonymous questionnaires were collected simultaneously. The oral fluids were tested with use of the Genscreen HIV $1 / 2$ version 2, (Bio-Rad) and Western Blot (Genlabs) diagnostic kits. The data analysis was performed using Stata version 8 . Saliva testing revealed HIV prevalence of $6.1 \%$ (21/349) among MSM in Slovakia. 75\% of HIV-positive cases were undiagnosed. The high-risk behaviour of MSM in respect to the number of their sexual partners as well as drug and condom usage did not correspond with their relatively high knowledge about HIVIAIDS. People's attitude towards MSM was found to be the worst in the religious context. Though our results reflect a relative good knowledge of MSM about HIVIAIDS infection, new HIV-positive cases and high-risk behaviour still appear, suggesting the need for more effective HIV prevention among members of this high-risk group in Slovakia.
\end{abstract}

Key words: HIV, AIDS, MSM, surveillance, risk behaviour

Address for correspondence: D. Staneková, NRC for HIVIAIDS prevention, Slovak Medical University, Limbová 14, 83301 Bratislava, Slovakia. E-mail: danica.stanekova@szu.sk

\section{INTRODUCTION}

HIV infection remains an important public health issue in Europe, although according to UNAIDS the number of newly diagnosed HIV positive patients is declining. There were approximately 2.6 million (2.3-2.8 million) people who became newly infected with HIV in 2009. This number is about one fifth (19\%) smaller than 3.17 million (2.9-3.4 million) people who were newly infected in 1999, and more than one fifth (21\%) smaller than in 1997, when annual new infections peaked (1). There is a strong evidence of resurgent HIV epidemics among men who have sex with men (MSM) in North America and in Western Europe and this group continues to represent a population at high risk of HIV infection $(2,3)$. Data from 23 western countries show that the annual number of men who have sex with men diagnosed with HIV infection rose by $86 \%$ between 2000 and 2006 (4). National surveillance data from 2000-2005 show significant increase in HIV newly diagnosed cases among MSM in Australia, Canada, Germany, the Netherlands, Spain, and the United States of America, including those with recently acquired and acute infection (5). Central Europe MSM group is also a vulnerable group due to their high-risk sexual behaviour (6). In the past decade, the number of syphilis cases and other sexually transmitted infections (STI) including HCV among MSM has also increased in Western European countries $(7,8)$. In Eastern Europe and Central Asia unprotected sex between men is responsible for less than $1 \%$ of newly diagnosed HIV positive patients for whom the route of transmission was identified (9). HIV prevalence is $1 \%$ or higher in two countries in this region, the Russian Federation and Ukraine, which together account for almost $90 \%$ of newly reported HIV cases (10).
Slovakia, a country bordering Ukraine, belongs to the countries with relatively low HIV prevalence. As of December 2010, there were 343 HIV-infected Slovaks and 114 foreigners registered in SR. About $65 \%$ of cases were infected by unprotected MSM intercourse (Staneková, personal information). Nevertheless, official data may underplay the actual extent of infection in this highly stigmatized population. Therefore, the EU project SIALON "Capacity building in HIV/Syphilis prevalence estimation using noninvasive methods among MSM in Southern and Eastern Europe" was carried out in 2008-2009 in seven EU countries. Its aim was to clarify the HIV prevalence in MSM in Europe using the Second Generation Surveillance System (SGSS), which combines monitoring of newly diagnosed HIV cases and indicators of sexual behaviour among persons in groups who are at the highest risk for infection. The goal of the EU project SIALON (2008-2009) was to gather reliable information on HIV prevalence among MSM in Southern and Eastern Europe. The 2nd generation surveillance of HIV infection was carried out in several towns of six EU countries, mostly in capitals, and preliminary data were published in the paper of Mirandola et al. in 2009 (11). The aim of our study was to provide deeper analysis of the results obtained at the end of the EU project SIALON in Slovakia.

\section{MATERIALS AND METHODS}

All methods used in the study are described in more detail in the paper by Mirandola et al. (11). 


\section{Study Design}

The study was a descriptive multi-centre biological and behavioural cross-sectional survey carried out in Slovakia and in additional six Southern and Eastern European countries: Greece, Spain, Romania, Slovenia, the Czech Republic, and Italy, mostly in capital cities.

Ethics Committee approval was obtained and an informed consent form was collected from each respondent. The oral fluid samples and the questionnaires were collected anonymously and a barcode was used to link behavioural and biological information. Respondents interested in getting their test results were informed that the test result was not meant to be diagnostic and for this reason, they should be tested again in line with international/ national guidelines.

\section{Study Population}

Participants from various MSM groups were tested by TLS method (time-located sampling) according to the following four criteria: having had sex (any kind of sex: oral and anal, penetrative or not) at least once with another man during the past 12 months before the study; having signed a written informed consent form; having agreed to answer the study questionnaire; having accepted to donate an oral fluid sample. Three exclusion criteria were adopted: age below 18 years; currently active injecting drug use (IDU) and having already participated in the study.

\section{Sampling}

Time-location (or time-space) sampling (TLS) was used to recruit representative samples. The sample size estimation study was calculated based on previous prevalence estimation studies when available (12). Four hundred persons were included in the planned survey in each country, separately.

\section{Data Collection and Questionnaire}

A self-administered pen-and-paper questionnaire taking into account UNGASS indicators $(10,13)$ was used to obtain information on the social, cultural and environmental context of respondents, access and barriers to voluntary counselling and testing (VCT), behavioural data on sex practices, risk-reducing strategies, condom use, STI history, self-reported/perceived serostatus and type of a partner. Steady partner was defined as a person with whom he/she was committed to and had sex, not necessarily meaning that the person was exclusively monogamous.

\section{Oral Fluid Sampling and Testing}

To collect oral fluids, the Oracol oral fluid collection kits (Malvern Medical Developments, Worcester, UK) were used. After collection, oral fluid samples were kept refrigerated and sent to the National reference centre for HIV/AIDS in Bratislava no more than 72 hours after collection. Subsequently they were sent to the Teaching Hospital-University of Verona, Immunology Unit in Verona, Italy. EIA testing Genscreen HIV $1 / 2$ version 2, (Bio-Rad) on oral fluid sample was performed according to the manufacturer's instructions. All positive samples were confirmed with the Western Blot test (Genlabs). Total antibodies
ELISA test was performed in order to assess sample suitability for testing.

\section{Enrolment}

Professionally trained workers from the Association of AIDS Help in Slovakia collected both self-administrated anonymous questionnaires and oral fluid samples. The enrolment period was nine months.

\section{Data Analysis}

Data analysis was performed using Stata version 8. Chi-square test was used for bivariate comparisons, and multivariate logistic regression was applied to assess odds ratios (OR) and significance of independent variables for main sexual behaviour outcomes.

\section{RESULTS}

\section{Study Population}

At the end of the study in Slovakia a total of 396 oral fluid samples and 387 questionnaires were collected.

The median age of respondents was 28 years (17-64 years). Most of the respondents $(354 / 387,91.4 \%)$ lived in Slovakia and declared Slovak nationality $(341 / 387,88.1 \%)$.

From 387 respondents 209 (54.0\%) and 145 (37.5\%) declared their most recent qualification as secondary school and university degree, respectively. More than half of 385 respondents (64.2\%) lived in a city (more than 100,000 inhabitants), less in smaller places like small town $(21.8 \%)$, village $(9.4 \%)$ and countryside (4.7\%). Most of 386 respondents lived with male partners (29.3\%), less with their parents $(28.8 \%)$, with their friends $(28.5 \%)$ or alone $(8.8 \%)$, and $(4.7 \%)$ in a heterosexual family (female partner and/ or children). Those living in a city lived significantly more often with a male partner and/or friends than those living in other small places $(p<0.001)$. MSM in cities also significantly less often lived with parents and relatives $(p=0.001)$. Concerning the sexual orientation, $84.7 \%$ of 386 respondents self-identified themselves as homosexual, $13.7 \%$ as bisexual and $0.5 \%$ as heterosexuals. As far as the proportion of MSM reached with HIV prevention programmes is concerned (UNGASS indicator 9), while excluding those offered by the project SIALON, in the last 12 months condoms have been given for free to $29.7 \%$ of $356,21.6 \%$ of $360,9.5 \%$ of 346 and $4.8 \%$ of 349 persons in a free distribution in saunas, clubs or discos, by a gay association, by other associations, or by a health service/clinic, respectively. According to the respondents experience people's attitude towards homosexuals or bisexuals was the worst in religious $88.5 \%$ (348/393) context comparing to others: friends $2.73 \%$ (10/366), parents $26.7 \%(98 / 367)$, work 23.8\% (90/378), school 52.29\% (194/371), political 72.22\% $(268 / 366)$ and gay scene $5.13 \%(19 / 370)$.

\section{HIV Prevalence and HIV/STI Testing}

In order to monitor HIV testing uptake, UNGASS indicator number 8 was used, which comprises the percentage of MSM tested for HIV over the last 12 months who also collected the result 
of their last HIV test. In the past 12 months, 147 of $381(38.6 \%)$ of respondents have had an HIV test, of which 81 (55.4\%) and 5 $(1.3 \%)$ were found HIV-negative and HIV-positive, respectively. Significantly more participants living in small places have never been tested for HIV comparing to those living in a city $(p=0.001)$. There were no significant differences in HIV-positive results between these two groups. Saliva testing revealed HIV prevalence of $6.1 \%(21 / 349)$ among MSM (UNGASS indicator 23). From these 21 MSM 5 were found HIV-positive during the last HIV test (Table 1).

Participants also declared that in the last 12 months, they have been diagnosed with one of the following sexually transmitted diseases: syphilis $(0.8 \%)$, gonorrhoea $(1.1 \%)$, chlamydia $(3.4 \%)$, uretritis $(5.3 \%)$, anogenital warts $(2.1 \%)$, hepatitis B $(1.1 \%)$, and genital herpes $(1.1 \%)$, while no differences were found between the answers of the respondents living in the cities and in smaller municipalities.

\section{Behavioural Data \\ Risk Behaviour over the Last 6 Months}

Most of respondents declared to have 1-4 steady or causal partners in the last six months (Table 2). Sex with steady partners vs. causal was declared by $277(73.08 \%)$ vs. $274(70.8 \%)$ of 379 men, respectively, while 147 (37.7\%) respondents had sex with both steady and casual partners. In our study, 99 (26.7\%) vs. 4 of 370 respondents $(1.1 \%)$ have had $2-5$ vs. more than 50 female sexual partners, respectively.

Participants reported following psychoactive and recreational drug use before or during sex: alcohol (83.63\%), viagra (10.46\%), poppers $(34.71 \%), 6.94 \%$ ecstasy, amphetamine $(4.65 \%)$, cocain $(5.64 \%)$, and others $(57.4 \%)$.

Table 1. HIV status of respondents

\begin{tabular}{|l|c|c|c|}
\hline HIV results & \multicolumn{3}{|c|}{ According to saliva testing } \\
\hline $\begin{array}{l}\text { According to } \\
\text { responders }\end{array}$ & HIV - & HIV + & Total \\
\hline HIV - & 266 & 9 & 275 \\
\hline HIV + & 3 & 5 & 7 \\
\hline Not known & 77 & 7 & 84 \\
\hline Total & 346 & 21 & 366 \\
\hline
\end{tabular}

Consistent condom use during the receptive vs. insertive anal sex with a steady and casual partner was declared by $13.14 \%$ vs. $14.44 \%$ and $20.3 \%$ vs. $25.56 \%$ of respondents, while any condom use by $19.06 \%$ vs. $17.63 \%$ and $5.62 \%$ vs. $6.67 \%$ of respondents, respectively (Table 3). Additionally, condom use during the receptive vs. insertive oral sex with a steady and causal partner was declared by $0.7 \%$ vs. $2.5 \%$ and $2.6 \%$ vs. $3.0 \%$ of participants, while any condom use by $49.5 \%$ vs. $41.9 \%$ and $26.6 \%$ vs. $28.8 \%$ of participants, respectively. Additionally, 187 of 286 (65.3\%) of respondents did not know HIV status of their male steady partner, while $5(1.7 \%)$ knew that their steady partner was HIV-positive. As far as the risk behaviour of these 5 persons is concerned, 2 of them had receptive and 1 had insertive anal sex with a condom sometimes, 1 was sometimes penetrated without a condom, and 2 had often and 1 sometimes insertive or receptive oral sex with their partners without a condom, respectively. Additionally, 3 men declared that they took drugs, mostly alcohol and poppers, before or during the sexual encounter.

On the question: "In the last 6 months, how have you met your male sexual partners?" the numbers of answers were as follows: through internet profiles or chat-lines - 178 (46.0\%); through newspaper advertisements $-5(1.3 \%)$; in the bar, pub, disco or the club - 122 (31.5\%); in sauna/dark room - 88 (22.7\%); in cruising settings (parks, public toilets, outdoor settings, beach) -14 $(3.6 \%)$; in gay society or through their friends $-85(22.0 \%)$; and in porno cinema - $18(4.7 \%)$.

\section{Risk Behaviour during the Last Sex}

In our study, 29.7\% (111/374) of participants used condom during their last sexual intercourse (UNGASS 19), 179 (47\%) of respondents had last sex with a steady partner while 173 $(45.4 \%)$ with a casual and $15(3.9 \%)$ with both or other partners 14 (3.7\%). Additionally, 202 (53.0\%) of respondents did not know HIV status of their last sexual partner, while 4 (1.0\%) knew that their last steady partner was HIV-positive. As far as the risk behaviour of these 4 persons is concerned, 2 penetrated their partners with a condom and 3 vs. 1 had insertive vs. receptive oral sex with their partners without a condom, respectively. Additionally, 2 respondents, who had oral sex without condom, declared, that they used some drugs before or during the last sexual encounter. None of the respondents had anal sex without condom. No differences were observed in risk behaviour mentioned above between participants living in the large cities and/ or smaller places in Slovakia.

Table 2. Number of steady and causal partners of respondents in the last six months

\begin{tabular}{|l|c|c|c|c|}
\hline \multirow{2}{*}{ Partners by age } & \multicolumn{2}{|c|}{ Steady partners } & \multicolumn{2}{c|}{ Causal partners } \\
\cline { 2 - 5 } & Respondents (N) & Respondents (\%) & Respondents (N) & Respondents (\%) \\
\hline 0 & 13 & 4.70 & 11 & 4.00 \\
\hline 1 & 154 & 55.60 & 46 & 16.80 \\
\hline $2-4$ & 91 & 32.80 & 117 & 42.70 \\
\hline $5-9$ & 15 & 5.50 & 50 & 18.20 \\
\hline $10-19$ & 4 & 1.40 & 34 & 12.50 \\
\hline $20-90$ & 0 & 0 & 16 & 5.90 \\
\hline Total & 277 & 100.00 & 274 & 100.00 \\
\hline
\end{tabular}


Table 3. Anal sex of respondents with steady partner over the last six months

\begin{tabular}{|c|c|c|c|c|c|c|c|c|c|c|}
\hline \multirow{2}{*}{ High risk behaviour } & \multicolumn{2}{|c|}{ Never } & \multicolumn{2}{|c|}{ Sometimes } & \multicolumn{2}{|c|}{ Often } & \multicolumn{2}{|c|}{ Always } & \multicolumn{2}{|c|}{ Total } \\
\hline & $\mathrm{N}$ & $\%$ & $\mathrm{~N}$ & $\%$ & $\mathrm{~N}$ & $\%$ & $\mathrm{~N}$ & $\%$ & $\mathrm{~N}$ & $\%$ \\
\hline $\begin{array}{l}\text { Been penetrated without } \\
\text { a condom }\end{array}$ & 116 & 41.73 & 77 & 27.70 & 32 & 11.51 & 53 & 19.06 & 278 & 100.00 \\
\hline $\begin{array}{l}\text { Penetrated your partner without } \\
\text { a condom }\end{array}$ & 126 & 45.32 & 74 & 26.62 & 29 & 10.43 & 49 & 17.63 & 278 & 100.00 \\
\hline Been penetrated with a condom & 147 & 53.65 & 71 & 25.91 & 20 & 7.30 & 36 & 13.14 & 274 & 100.00 \\
\hline $\begin{array}{l}\text { Penetrated your partner with } \\
\text { a condom }\end{array}$ & 149 & 53.79 & 69 & 24.91 & 19 & 6.86 & 40 & 14.44 & 277 & 100.00 \\
\hline
\end{tabular}

\section{Risk Behaviour of HIV-positive MSM}

In our study, 21 persons were found HIV-positive in saliva testing. Their risk behaviour over the last 6 months was as follows: 6 had 3 and more sexual partners; 6 never did use condom during the oral sex; 2 always and 6 sometimes ejaculated into the mouth of their partner. None of the HIV-positive MSM declared usage of condom during active oral sex with their steady partner. Seven infected persons had passive oral sex with their steady partner without condom often, and just seven respondents always used condom during anal sex with their steady partner. During the oral sex with causal partner 7 MSM never used condom and just 2 used it always. Two vs. four men never used condom during the receptive vs. insertive anal sex with causal partner, respectively. Due to the relatively small number of HIV-positive MSM found in our study, precise comparative analysis of risk behaviour with the HIV-negative group was not available.

\section{Knowledge about HIV}

In our study, $89.3 \%(341 / 382)$ of participants knew where they could go to get an HIV test and $11.9 \%$ (46/385) vs. $12.2 \%$ (47/385) of respondents declared that having sex with only one steady, uninfected partner reduce vs. does not reduce the risk of HIV transmission, respectively. The rest of the respondents -292 , did not know the answer. From the group of 385 respondents, 360 $(93 \%)$ vs. $9(2.3 \%)$ thought vs. did not think that using a condom can reduce the risk of HIV transmission, respectively. Additionally, $91.1 \%(350 / 385)$ vs. $2.9 \%$ (11/385) participants agreed vs. did not agree with the sentence: "A healthy-looking person can be HIV-positive.", respectively.

\section{DISCUSSION}

The majority of respondents of the study were recruited in Bratislava. Our study revealed a total of $6.1 \%$ HIV prevalence among MSM. In SIALON I the HIV prevalence has ranged from $2.5 \%$ in Prague, 5.1\% in Ljubljana, 6.4\% in Bucharest, $11.8 \%$ in Verona to $17.0 \%$ in Barcelona (11). Additionally, in other small surveys, the HIV prevalence among men who have sex with men has ranged from zero in Belarus, Lithuania and parts of Central Asia to $5 \%$ in Georgia (14), 6\% in the Russian Federation (15) and between 4\% (in Kyiv) and 23\% (in Odessa) in Ukraine (16). The 3,160 new HIV diagnoses among men who have sex with men in 2007 in the United Kingdom were the most ever reported up to that point (17). In our study, HIV prevalence in MSM was higher than that described in 1996 similar study among MSM in Slovakia (18).

In the last 12 months, about one third of our study respondents had an HIV test. Similar results were found in the MSM group in Ljubljana while in Prague, Bucharest, Barcelona and Budapest this percentage was higher (11). Our results also revealed the fact that just about one fourth of HIV-positive participants knew they were infected. Similarly, in the study from Scotland, $4.4 \%$ of MSM were found HIV-positive, from them $41.7 \%$ were undiagnosed. More than half had a negative HIV test result and perceived themselves to be HIV-negative (19). Study from New Zealand confirmed that one fifth (20.9\%) of HIV infected men were undiagnosed whereas HIV prevalence in MSM was 6.5\% (20).

HIV infection could be often associated with other STI. In our study, $0.8-3.4 \%$ of participants suffered from other STI in the past. In the study conducted in Spain, $64.5 \%$ of MSM reported having sexually transmitted infection in their lifetime, and $4.8 \%$ and $3.3 \%$ reported having been diagnosed with gonorrhoea and syphilis during the previous year, respectively (21). During the period from 2000-2005, of 3.3\% HIV diagnoses in Europe also increased in primary and secondary syphilis diagnoses occurring among MSM, but recent HIV testing among MSM did not seem to confirm the increase (5).

The fear of being stigmatized can compel many men who have sex with men to also have sexual relationships with women. In our study, participants self-identified themselves more often as homosexual (84.7\%) and as bisexual (13.7\%) than those involved in the previous study realized in Slovakia in 1996 (Staneková et al., 2000). Despite of this fact $26.7 \%$ and $1.1 \%$ participants declared that they have had 2-5 and more than 50 female sexual partners, respectively. Similarly, during the 12-month study period in Denmark, $88 \%$ of the respondents had sex with men exclusively, whereas $12 \%$ had sex with both men and women, respectively. Interestingly, bisexual behaviour was stated by $17 \%$ of the internet respondents, but only by $4 \%$ of those who responded to paper questionnaires (22). A study from Spain (21) shows that most men reported also that their sexual orientation was homosexual $(89.2 \%)$ and $8.5 \%$ characterized themselves as bisexual. Other behavioural studies also suggest that African MSM often have sex with women and indicate their potential for transmitting HIV to female population (23).

Similarly to other studies $(11,22)$, two thirds of the respondents in our study lived in areas with more than 100,000 inhabitants. Those living in a city lived significantly more often with male partner and/or friends than those living in other small places: MSM in cities also significantly less often lived with parents and 
relatives. This fact could be due to the bad respondents' experience with people's attitude towards homosexuals or bisexuals in religious, mostly catholic, context notable especially in small towns and villages in Slovakia. Similarly, the study from Spain revealed that $7.5 \%$ and $11.0 \%$ of homo/bisexuals had a high degree of internalized homophobia and had experienced insults and/or attacks in the previous years because of their sexual orientation, respectively (21).

In our study, high level of psychoactive and recreational drug use before or during sex was reported by MSM. Similarly, a high prevalence of drug use before or during sexual intercourse in the previous 12 months was described in the study from Spain. The most widely used drugs were poppers, cannabis, and cocaine (40.8\%, 26.0\% and $18.8 \%$, respectively); $28.6 \%$ consumed two or more different drugs (21).

In our study, just about one third of participants used condom during their last sexual intercourse. Similar results were found in the MSM group only in Prague, while in Ljubljana, Bucharest, Barcelona and Budapest this percentage was higher (11). The increase in MSM higher-risk sexual behaviour is remarkably associated with the increased incidence of HIV infection. For example, in the United States of America, new HIV infections attributed to unprotected sex between men increased by more than 50\% from 1991-1993 to 2003-2006 (24). Similar trends have been reported in Canada (25). Our study revealed that almost half of the participants reported having had sex with 1 steady and 2-4 causal partners during the last 6 months and $37.7 \%$ had both steady and causal partners. In Denmark, 34\% of MSM had only casual partners (one or more) during the last 12 months. A similar proportion, $35 \%$, had both steady and casual partners, whereas only $20 \%$ had one steady partner. A small group (2\%) reported more than one steady partner but no casual partners (22). Researchers in Catalonia (Spain), for example, reported that one third (32\%) of MSM have recently had unprotected anal sex with a casual partner (21) and surveys conducted in Denmark and Scotland have reported similar findings $(22,26)$.

Our study described the evidence of the ongoing risk practices among most of MSM with HIV infection. The review of 53 cross-sectional and longitudinal surveys of HIV-positive MSM and MSM of mixed HIV status published after 2000 indicates that HIV-positive MSM have adopted risk reduction strategies, but roughly two in five HIV-positive MSM continue to engage in unprotected anal intercourse (UAI), which represents a risk for continued HIV and STI (sexually transmitted infection) transmission (27). Risk practices of MSM in our study did not correspond with their relatively satisfactory knowledge of HIV prevention. Similarly, risk behaviour was high and knowledge of HIV transmission methods was low among MSM in Kuala Lumpur (28) as well as in Heilongjiang province in China, where the prevalence of HIV infection was found to be low among MSM, and the fact that the risk behaviours were frequent in the population was alarming (29).

\section{CONCLUSIONS}

This is the first estimate of actual and undiagnosed HIV infection among a community sample of MSM in Slovakia. Whereas results of our study among MSM reflect their relatively good knowledge of the risks associated with HIV/AIDS infection, the revelation of new HIV-positive cases linked with high-risk behaviour suggests the need for more effective HIV prevention among MSM in Slovakia. The prevention should focus on raising condom use and earlier diagnosis among those who are most at risk, and encouraging safe behaviour after being diagnosed. Additionally, prevention efforts targeting HIV-positive MSM with the aim to assist them in adopting and maintaining safer sexual behaviour need to be intensified.

\section{Acknowledgement}

We thank for the collaboration to Mirandola M, Folch Toda C, Jedlicka J, Krampac I, Nita I, Stehlikova D, Toskin I, Gios L, Foschia JP, Breveglieri M, Furegato M, Castellani E, Bonavina MG from EU project SIALON that was founded by the European Commission under the European Public Health Programme 2003-2008 (Grant No. 2007 309).

\section{Conflict of Interests}

None declared

\section{REFERENCES}

1. UNAIDS. Global report: UNAIDS report on the global AIDS epidemic, 2010. UNAIDS; 2010

2. Bezemer D, de Wolf F, Boerlijst MC, van Sighem A, Hollingsworth TD, Prins M, et al. A resurgent HIV-1 epidemic among men who have sex with men in the era of potent antiretroviral therapy. AIDS. 2008 May 31;22(9):1071-7.

3. van de Laar MJ. HIV/AIDS and other STI in men who have sex with men - a continuous challenge for public health. Euro Surveill. 2009 Nov 26;14(47). pii: 19423.

4. Likatavicius G, Klavs I, Devaux I, Alix J, Nardone A. An increase in newly diagnosed HIV cases reported among men who have sex with men in Europe, 2000-6: implications for a European public health strategy. Sex Transm Infect. 2008 Nov;84(6):499-505.

5. Sullivan PS, Hamouda O, Delpech V, Geduld JE, Prejean J, Semaille C, et al.; Annecy MSM Epidemiology Study Group. Reemergence of the HIV epidemic among men who have sex with men in North America, Western Europe, and Australia, 1996-2005. Ann Epidemiol. 2009 Jun;19(6):42331.

6. van de Laar MJ, Likatavicius G. HIV and AIDS in the European Union, 2008. Euro Surveill. 2009 Nov 26;14(47). pii: 19422.

7. Savage EJ, Hughes G, Ison C, Lowndes CM; European Surveillance of Sexually Transmitted Infections network. Syphilis and gonorrhoea in men who have sex with men: a European overview. Euro Surveill. 2009 Nov 26;14(47). pii: 19417.

8. Urbanus AT, van de Laar TJ, Stolte IG, Schinkel J, Heijman T, Coutinho RA, et al. Hepatitis C virus infections among HIV-infected men who have sex with men: an expanding epidemic. AIDS. 2009 Jul 31;23(12):F1-7.

9. van de Laar MJ, Likatavicius G, Stengaard AR, Donoghoe MC. HIV/ AIDS surveillance in Europe: update 2007. Euro Surveill. 2008 Dec 11;13(50). pii: 19066.

10. UNAIDS. Hidden HIV epidemic amongst MSM in Eastern Europe and Central Asia [Internet]. Geneva: UNAIDS; 2009 [cited 2010 Oct 17]. Available from: http://www.unaids.org /en/ Knowledge Centre/ Resources/FeatureStories / archive / 2009 / 20090126_MSM Ukraine.asp.

11. Mirandola M, Folch Toda C, Krampac I, Nita I, Stanekova D, Stehlikova D, et al.; SIALON network. HIV bio-behavioural survey among men who have sex with men in Barcelona, Bratislava, Bucharest, Ljubljana, Prague and Verona, 2008-2009. Euro Surveill. 2009 Dec 3;14(48). pii: 19427.

12. Folch C, Casabona J, Muñoz R, Zaragoza K. Trends in the prevalence of HIV infection and risk behaviors in homo- and bisexual men. Gac Sanit. 2005 Jul-Aug;19(4):294-301. (In Spanish.)

13. Monitoring the Declaration of Commitment on HIV/AIDS: guidelines on construction of core indicators: 2008 reporting [Internet]. Geneva: UNAIDS; 2007 [cited 2014 Aug 8]. Available from: http://www.unaids. org/sites/default/files/media_asset/jc1318_core_indicators_manual_en_0. pdf. 
14. Baral S, Sifakis F, Cleghorn F, Beyrer C. Elevated risk for HIV infection among men who have sex with men in low- and middle-income countries 2000-2006: a systematic review. PLoS Med. 2007 Dec;4(12):e339.

15. van Griensven F, de Lind van Wijngaarden JW, Baral S, Grulich A. The global epidemic of HIV infection among men who have sex with men. Curr Opin HIV AIDS. 2009 Jul;4(4):300-7.

16. Kruglov YV, Kobyshcha YV, Salyuk T, Varetska O, Shakarishvili A, Saldanha VP. The most severe HIV epidemic in Europe: Ukraine's national HIV prevalence estimates for 2007. Sex Transm Infect. 2008 Aug;84 Suppl 1:i37-i41.

17. Lattimore S, Yin Z, Logan L, Rice B, Thornton A, Molinar D, et al. Situation of HIV infections and STIs in the United Kingdom in 2007. Euro Surveill. 2008 Dec 4;13(49). pii: 19059.

18. Staneková D, Habeková M, Wimmerová S, Grambličková I. HIV infection and sexual behaviour among homosexual and bisexual men in Bratislava. Cent Eur J Public Health. 2000 Aug;8(3):172-5.

19. Williamson LM, Hart GJ. HIV prevalence and undiagnosed infection among a community sample of gay men in Scotland. J Acquir Immune Defic Syndr. 2007 Jun 1;45(2):224-30.

20. Saxton PJ, Dickson NP, Griffiths R, Hughes AJ, Rowden J. Actual and undiagnosed HIV prevalence in a community sample of men who have sex with men in Auckland, New Zealand. BMC Public Health. 2012 Feb 1;12:92.

21. Folch C, Munoz R, Zaragoza K, Casabona J. Sexual risk behaviour and its determinants among men who have sex with men in Catalonia, Spain. Euro Surveill. 2009 Nov 26;14(47). pii: 19415.

22. Cowan SA, Haff J. HIV and risk behaviour among men who have sex with men in Denmark - the 2006 Sex Life Survey. Euro Surveill. 2008 Nov 27;13(48). pii: 19050 .
23. Smith A, Muhaari A, Agwanda C, Kowuor D, van der Elst E, et al. Female clients and partners of MSM sex workers in Mombasa, Kenya. In: 17th Conference on Retroviruses and Opportunistic Infections; 2010 Feb 1619; San Francisco, California, USA.

24. Hall HI, Song R, Rhodes P, Prejean J, An Q, Lee LM, et al.; HIV Incidence Surveillance Group. Estimation of HIV incidence in the United States. JAMA. 2008 Aug 6;300(5):520-9.

25. Summary: estimate of HIV prevalence and incidence in Canada, 2008 Ottawa: Public Health Agency of Canada; 2009.

26. Hart GJ, Williamson LM. Increase in HIV sexual risk behaviour in homosexual men in Scotland, 1996-2002: prevention failure? Sex Transm Infect. 2005 Oct;81(5):367-72.

27. van Kesteren NM, Hospers HJ, Kok G. Sexual risk behavior among HIV-positive men who have sex with men: a literature review. Patient Educ Couns. 2007 Jan;65(1):5-20.

28. Kanter J, Koh C, Razali K, Tai R, Izenberg J, Rajan L, et al. Risk behaviour and HIV prevalence among men who have sex with men in a multiethnic society: a venue-based study in Kuala Lumpur, Malaysia. Int J STD AIDS. 2011 Jan;22(1):30-7.

29. Liu S, Wang K, Yao S, Guo X, Liu Y, Wang B. Knowledge and risk behaviors related to HIV/AIDS, and their association with information resource among men who have sex with men in Heilongjiang province, China. BMC Public Health. 2010 May 14;10:250.

Received April 15, 2013 Accepted in revised form August 11, 2014 